\section{P10.11 SERUM ANTIBODY RESPONSES FOLLOWING ANAL AND PENILE INFECTION WITH HUMAN PAPILLOMAVIRUS IN TEENAGE MEN WHO HAVE SEX WITH MEN}

${ }^{1,2,3} \mathrm{H}$ Zou ${ }^{*},{ }^{4,5,6} \mathrm{~S}$ Tabrizi, ${ }^{3} \mathrm{~A}$ Grulich, ${ }^{7} \mathrm{~J}$ Hocking, ${ }^{4,5,6} \mathrm{~S}$ Garland, ${ }^{1,8} \mathrm{C}$ Bradshaw ${ }^{1,8} \mathrm{C}$ Fairley ${ }^{\#},{ }^{1,8} \mathrm{M}$ Chen ${ }^{\#}$. ${ }^{1}$ Melbourne Sexual Health Centre, Alfred Health, Melbourne, Australia; ${ }^{2}$ School of Population and Global Health, University of Melbourne, Melbourne, Australia; ${ }^{3}$ Kirby Institute, University of New South Wales, Sydney, Australia; ${ }^{4}$ Department of Obstetrics and Gynaecology, University of Melbourne, Melbourne, Australia; ${ }^{5}$ Department of Microbiology and Infectious Diseases, Royal Women's Hospital, Melbourne, Australia; ${ }^{6}$ Murdoch Childrens Research Institute, Melbourne, Australia; ${ }^{7}$ Centre for Women's Health, Gender and Society, University of Melbourne, Melbourne, Australia; ${ }^{8}$ Central Clinical School, Monash University, Melbourne, Australia; \#Joint Last Authors

\subsection{6/sextrans-2015-052270.439}

Introduction No previous studies have measured HPV seroconversion rates following incident HPV infections of the anus. We examined these in teenage men who have sex with men (MSM). Methods A cohort of 200 MSM aged 16-20 years were assessed at baseline, 3, 6 and 12 months. At each visit penile and anal swabs were collected for HPV DNA and serum for HPV antibodies for types $6,11,16$ and 18. Seroconversion was defined as the detection of HPV antibodies following a negative antibody result for the same HPV genotype at baseline.

Results The seroincidence rates for HPV types 6, 11, 16 and 18 were: $18.8,6.7,3.9$, and 5.8 per 100 person-years respectively. Men who experienced incident anal HPV infections from types 6 or 11 were significantly more likely to develop serum antibodies to the same HPV type than those who experienced incident anal infections from types 16 and 18 (72.7\% vs $18.2 \%$, Odds ratio $(\mathrm{OR})=12.0,95 \%$ Confidence interval $(\mathrm{CI}): 2.5-58.1)$. However the likelihood to seroconvert did not differ between men who experienced incident penile HPV infections from types 6 or 11 and those who experienced incident penile infections from types 16 or $18(42.9 \%$ vs $20.0 \%$, OR $=3.0,95 \% \mathrm{CI}$ : 0.2-53.2). The median time between incident anal HPV infection and seroconversion for HPV 6, 11, 16, and 18 was: 91 days, 38 days, 161 days and 182 days respectively.

Conclusion Serum antibody responses were more likely to occur following anal infections with HPV types 6 and 11 than with types 16 and 18 . Seroconversion may occur at a variable number of months after anal HPV infection.

Disclosure of interest statement This investigator initiated study was funded by Merck. Merck had no input into the design, analysis or reporting of the study. CKF has received honoraria from CSL Biotherapies and Merck and research funding from CSL Biotherapies. CKF owns shares in CSL Biotherapies the manufacturer for Gardasil. JSH has received an honorarium from CSL Biotherapies and is an investigator on an Australian Research Council funded project (LP0883831) that includes CSL Biotherapies as a research partner. AEG has received honoraria and untied research funding from CSL biotherapies, and has received honoraria from Merck. SMG has received advisory board fees and grant support from CSL and GlaxoSmithKline, and lecture fees from Merck, GSK and Sanofi Pasteur; in addition, she has received funding through her institution to conduct HPV vaccine studies for MSD and GSK. SMG is a member of the Merck Global Advisory Board as well as the Merck Scientific Advisory Committee for HPV. None of this relates to this specific work. MYC reported his institution received a grant from Merck Sharp Dohme that supported the conduct of the study. MGL receives grants from Bristol Myer Squibb, Gilead, GlaxoSmithKline, Janssen-Cilag, Merck, Pfizer and Roche which are not related to this project. All other authors have no conflicts of interest.

\section{P10.12 ROLE OF CONDYLOMA ACUMINATA IN INCIDENT HIV INFECTION: A POPULATION-BASED COHORT STUDY IN TAIWAN 2000-2010}

${ }^{1,2} \mathrm{H}$ Zou* ${ }^{*}{ }^{2,3} \mathrm{Y}$ Chen, ${ }^{4} \mathrm{C}$ Strong, ${ }^{4} \mathrm{C} \mathrm{Li},{ }^{5,6} \mathrm{~W}$ Ko, ${ }^{2,5} \mathrm{~N} \mathrm{Ko}$. ${ }^{1}$ Kirby Institute, University of New South Wales, Sydney, 2052, Australia; ${ }^{2}$ Department of Nursing, National Cheng Kung University, Tainan, 701, Taiwan; ${ }^{3}$ Institute of Allied Health Sciences, College of Medicine, National Cheng Kung University, Tainan, 701, Taiwan; ${ }^{4}$ Department of Public Health, National Cheng Kung University, Tainan, 701, Taiwan; ${ }^{5}$ Department of Internal Medicine, National Cheng Kung University, Tainan, 701, Taiwan; ${ }^{6}$ College of Medicine, National Cheng Kung University, Tainan, 701, Taiwan

\subsection{6/sextrans-2015-052270.440}

Introduction The role of Condyloma Acuminata (CA) in incident HIV infection has not been well documented. We aimed to elucidate this relationship by analysing a large national cohort.

Methods Medical claim records 2000-2010 of 1 million individuals randomly selected from the Taiwan National Health Insurance Research Database (NHIRD, approximately 23 million individuals in total) were retrieved. We included all patients treated for CA (PWCs) and patients screened for and confirmed without CA (PWOCs). We matched five patients never screened for CA (PNCs) for every one PWC by year of first CA treatment/first clinical visit, gender and age. PWCs, PWOCs and PNCs were followed from the date of first CA treatment, first $\mathrm{CA}$ screening and first clinical visit, retrospectively. Endpoint was incident HIV infection. Chi-square test was used to compare socio-demographic characteristics among patients. Characteristics with a $\mathrm{P}$ value of $<0.1$ using univariate Cox regression were entered into a multivariate Cox regression model to calculate adjusted hazard ratio (aHR) of incident HIV infection.

Results We included 1539 PWCs, 1106 PWOCs and 7695 PNCs. The three groups were comparable with respect to age, gender, location and income. HIV incidence was $284.0(95 \%$ confidence interval (CI): 164.9-489.1), 110.6 (95\% CI: $41.5-$ 294.7) and 3.8 (95\% CI: 0.5-27.2) per 100,000 person-years among PWCs, PWOCs and PNCs, respectively. Compared to PNCs, PWCs were 66.4 (8.6-510.2) times and PWOCs 33.7 (3.8-303.5) times more likely to have incident HIV infection. Other variables associated with incident HIV infection were being a male $(\mathrm{aHR}=10.5,95 \% \mathrm{CI}$ : 2.4-46.1), being under 30 years of age ( $\mathrm{aHR}=3.5,95 \% \mathrm{CI}: 1.9-9.5)$ and syphilis diagnosis with treatment before HIV infection $(\mathrm{aHR}=4.5,95 \% \mathrm{CI}$ : 1.0-19.7).

Conclusion CA is associated with elevated incident HIV infection. HIV risk reduction interventions among patients with CA are warranted.

Disclosure of interest statement This study is funded by the Taiwan Ministry of Science (Project ID: 1030087343). All authors declare no conflict of interest.

\section{P10.13 PREVALENCE AND PREDICTORS OF HIGH-RISK ANAL HUMAN PAPILLOMAVIRUS (HPV) TYPES IN THE STUDY OF THE PREVENTION OF ANAL CANCER (SPANC)}

${ }^{1} \mathrm{IM}$ Poynten* ${ }^{*}{ }^{2} \mathrm{~S}$ Tabrizi, ${ }^{1} \mathrm{~F}$ Jin, ${ }^{1,3} \mathrm{DJ}$ Templeton, ${ }^{4} \mathrm{RJ}$ Hillman, ${ }^{2} \mathrm{~A}$ Cornall, ${ }^{2} \mathrm{~S}$ Phillips, ${ }^{2} \mathrm{SM}$ Garland, ${ }^{5} \mathrm{C}$ Fairley, ${ }^{1} \mathrm{AE}$ Grulich, on behalf of the SPANC Research Team. ${ }^{1}$ The Kirby Institute, University of New South Wales, Australia; ${ }^{2}$ Royal Women's Hospital, University of Melbourne, Melbourne, Australia; ${ }^{3}$ RPA Sexual Health, Sydney Local Health District, Sydney, Australia; ${ }^{4}$ Western Sydney Sexual Health Centre, University of Sydney, Australia; ${ }^{5}$ Melbourne Sexual Health Centre, Melbourne, Australia

10.1136/sextrans-2015-052270.441 